\title{
Digital Immunity Module: Preventing Unwanted Encryption using Source Coding
}

This paper was downloaded from TechRxiv (https://www.techrxiv.org).

\section{LICENSE}

CC BY-NC-SA 4.0

SUBMISSION DATE / POSTED DATE

04-01-2022 / 07-01-2022

\section{CITATION}

Mahboubi, Arash; Ansari, Keyvan; Camtepe, Seyit; Duda, Jarek; Morawiecki, Paweł; Pawłowski, Marcin; et al. (2022): Digital Immunity Module: Preventing Unwanted Encryption using Source Coding. TechRxiv. Preprint. https://doi.org/10.36227/techrxiv.17789735.v1

DOI

10.36227/techrxiv.17789735.v1 


\section{Digital Immunity Module: Preventing Unwanted Encryption using Source Coding}

\author{
Arash Mahboubi \\ Charles Sturt University \\ amahboubi@csu.edu.au \\ Paweł Morawiecki \\ Polish Academy of Sciences \\ pawel.morawiecki@gmail.com
}

\author{
Keyvan Ansari \\ University of the Sunshine Coast \\ kansari@usc.edu.au \\ Marcin Pawłowski \\ Polish Academy of Sciences \\ pawlowski.mp@gmail.com
}

\author{
Seyit Camtepe \\ Data61, CSIRO \\ seyit.camtepe@data61.csiro.au
}

Jagiellonian University

dudajar@gmail.com

Unwanted data encryption, such as ransomware attacks, continues to be a significant cybersecurity threat. Ransomware is a preferred weapon of cybercriminals who target small to large organizations' computer systems and data centres. It is malicious software that infects a victim's computer system and encrypts all its valuable data files. The victim needs to pay a ransom, often in cryptocurrency, in return for a decryption key. Many solutions use methods, including the inspection of file signatures, runtime process behaviors, API calls, and network traffic, to detect ransomware code. However, unwanted data encryption is still a top threat. This paper presents the first immunity solution, called the digital immunity module (DIM). DIM focuses on protecting valuable business-related data files from unwanted encryption rather than detecting malicious codes or processes. We show that methods such as file entropy and fuzzy hashing can be effectively used to sense unwanted encryption on a protected file, triggering our novel source coding method to paralyze the malicious manipulation of data such as ransomware encryption. Specifically, maliciously encrypted data blocks consume exponentially larger space and longer writing time on the DIM-protected file system. As a result, DIM creates enough time for system/human intervention and forensics analysis. Unlike the existing solutions, DIM protects the data regardless of ransomware families and variants. Additionally, DIM can defend against simultaneously active multiple ransomware, including the most recent hard to detect and stop fileless ones. We tested our solution on 39 ransomware families, including the most recent ransomware attacks. DIM successfully defended our sample file dataset (1335 pdf, jpg, and tiff files) against those ransomware attacks with zero file loss.

Index Terms-Ransomware, Malware, Malicious Cryptography, Information Theory, File System, Fileless ransomware.

\section{INTRODUCTION}

Cryptoviruses, which are widely referred to as ransomware, are the most common unwanted data encryption techniques. The terms ransomware and cryptovirus are interchangeably used in the literature when referring to malware that uses cryptography to make data inaccessible. Ransomware attacks are not a new menace, though. The core concept of ransomware and its first implementation were published as early as 1989 [1]. Since then, they have evolved significantly, becoming more contagious and sophisticated. The new generation of ransomware employs a wide range of symmetric, asymmetric, and hybrid cryptographic algorithms, elevating the generation, exchange, and storage of cryptographic keys to a highly advanced level. This renders decryption of data impossible without paying a ransom for the decryption key. Moreover, it is not unusual for malware designers to deploy home-brewed cryptosystems to evade malware detection. Such detection tries to identify suspicious functionalities and abnormal behaviours, such as encryption, by looking at cryptographic API calls [2].

Machine Learning (ML) based techniques are the most current trend to detect ransomware before it encrypts valuable files on the infected computer [3]. A majority of published ML-based detection solutions focus on API calls, abnormal behavior and white lists. Despite great success in detecting known ransomware, malware authors continuously adapt their design strategies to bypass ML-based techniques. For exam- ple, they hide ransomware within neural network models to camouflage ransomware characteristics [4].

Ransomware is becoming a cat-and-mouse game with attack vectors outpacing the countermeasures. No one-size-fits-all solution that can detect/stop all types of ransomware has been yet proposed, while continually emerging ransomware families and variants defeat existing countermeasures and make them inadequate. We observe that when a computer is infected by one or more ransomware, only a relatively small portion of the files can not be recovered through a re-image of the computer. It is possible to separate those critical files from the rest of the operating system and focus only on those critical files. This transforms the problem from detecting a ransomware process in the user space of an operating system to protecting a set of critical data files in storage using a kernellevel module. This brings a level of resilience against the variations between ransomware families and family members, including fileless ransomware. Hence, we propose a kernelmode level solution, i.e., file system firmware that takes over the role of transferring data buffer by buffer to and from the storage. Our solution detects maliciously encrypted buffers (i.e., unwanted encryption) and accordingly stops/slows down ransomware activities on the file system.

This paper presents a novel mechanism for file-system level protection of critical data files against unwanted ransomware encryption, called Digital Immunity Module (DIM). DIM sits between a local file system and a network file-sharing system, 
where sensitive and ransom-worthy files are stored. The critical data files include *.doc, *.pdf, *.jpg, *.tiff, etc. DIM detects and prevents malicious file write activities. DIM utilizes fuzzy file signature indexing and reacts to entropy changes that are caused by malicious encryption in progress. It stops or slows down malicious encryption by expanding the encrypted files exponentially and provides sufficient time for preventive measures.

The main contributions of this paper are: (1) Investigation of both the workflow and modus operandi of a wide range of published ransomware families and variants, including recent attacks. The investigation is essential in order to understand how ransomware encrypts and writes encrypted data files to storage; (2) Development of a novel kernel-mode algorithmic approach that can detect whether or not write buffers are being maliciously encrypted; (3) Development of a novel encoding module based on an exploited lossless data compression (source coding) scheme which reacts to unwanted encryption of write buffers by growing (i.e., exploited compression) them into larger sizes at GB (TB in theory) scales. This reactive size increase in write buffers can either slow down the ransomware or paralyze the system (or a set of processes operating on the protected volume) to allow sufficient time for intervention by mitigation solutions or human operators; (4) Design of a user-mode solution, which rescues attacked critical files. The solution is implemented as a digital immunity module and has been tested against 39 ransomware families and variants, including the most recent ransomware. Furthermore, to the best of our knowledge, DIM is the only effective way to combat fileless ransomware.

The rest of the paper is organized as follows. Section $\Pi$ discusses a threat model and a modus operandi of ransomware. Section III presents our proposed digital immunity module (DIM). In Section IV, we describe novel DIM algorithms and modules. In Section $\mathrm{V}$, we evaluate the operation of the digital immunity module and present the evaluation results. In Section VI. we compare DIM with another current model. Finally, Sections VII and VIII present related works, a summary of our work and future research directions.

\section{Threat Model}

Our threat model has been developed after careful analysis of a wide range of published ransomware variants that use many distinct algorithms and methods to encrypt data on network storage technologies [5]. Network storage technologies may have many point-to-point connections within the network. Many servers connect to storage arrays and appliances that support several different operating environments and applications. Data is vulnerable to exposure as long as they are stored on a network system. In general, ransomware infections can be initiated by spam, phishing websites, macros, backdoors, and flash drives [6]. We suppose that clients and hence network shared drives are potentially vulnerable to ransomware attacks. In our threat model, we assume that an adversary (1) discovers unknown vulnerabilities (e.g., zero-day) and exploits them to deploy their ransomware, (2) may use any possible attack vectors to initiate ransomware infection (or infections), (3) has full knowledge of the DIM design and its detection and prevention mechanisms, (4) targets critical business data on shared drives by infecting vulnerable user computers with write access right to these shared drives, (5) aims to encrypt data using ransomware in a revertible way to secure ransom revenue (i.e., no evidence for victims to believe that they won't recover their data after ransom is paid). The last point will motivate attacker to keep data till it is encrypted into a revertible form.

Further, let the following statements be true: (1) Protecting business data files is prioritized over service functionality, usability and performance during a ransomware attack. (2) Data encryption and compression are assumed to happen after DIM and before disk I/O to expose DIM unencrypted and uncompressed files only. (3) DIM does not protect the entire system, including the OS data files. A computer re-image can recover these files (i.e., files other than protected data files).

In preparation for our comprehensive study described in the paper, we pose the following questions. (1) How can DIM detect and identify encrypted write buffers independent of the encryption algorithm used? (2) How can DIM obstruct the functionality of ransomware? (3) Where can DIM be embedded in a resilient system?

\section{A. Modus Operandi of Ransomware}

Ransomware is a malicious software normally installed by an unsuspecting user who is tricked by an adversary to download it. For example, a ransomware may include encryption algorithms such as AES, ChaCha, and/or RSA, a random cryptographic key $K$ and its encrypted version $E_{p k}(K)$, where $p k$ is a public key of the adversary.

Once a ransomware program has been installed on a local machine, it encrypts files stored on the user's HD. Though variations may exist, in general, a ransomware fetches a file $f$ to RAM and encrypts it using the key $K$. Next, it stores the encrypted file $E_{K}(f)$ on HD and, at the same time, removes the plaintext file $f$ from it. It continues until all files are encrypted, and their plaintext copies are removed from HD. The key $K$ is then discarded, but its encrypted copy $E_{p k}(K)$ is stored on HD. Finally, the ransomware displays a message asking the user to pay for the key $s k$ that allows to recover the key $K$ from $E_{p k}(K)$. Once the e-payment is received, the owner of the ransomware releases the secret key $s k$ that matches the public key $p k$. This allows the user to recover the key $K$ from $E_{p k}(K)$ and decrypt the files stored on HD.

For example, LockerGoga is a relatively new, advanced ransomware family that targets businesses using a compromised Microsoft PsExec system management tool. LockerGoga made its debut when it was allegedly involved in an attack on an engineering consulting firm in France. Based on our analysis, LockerGoga is, by far, one of the most sophisticated ransomware with a wide range of malicious features. A fully loaded copy of LockerGoga contains all the necessary libraries and routines, including cryptographic functions and virtual environments. The ransomware family is exceptional in terms of sophistication, especially when compared to other ransomware families. At the initial stage of an attack, LockerGoga locks 
out a system administrator, disables all network interfaces and takes control over all processes. Note that once this is done, network intrusion detection tools are unable to react to the attack. The LockerGoga package includes a comprehensive library of modular signatures and symmetric-key primitives built on top of the Ursa library.

Furthermore, ransomware developers are now employing advanced techniques such as "fileless" in which ransomware (e.g., netwalker.B) is either embedded in a native scripting language or written directly into memory, without even requiring to be written into HD. This poses a significant challenge to existing ransomware detection methods. DIM successfully defends data files against fileless ransomware. DIM is able to recover files even when ransomware copies files into memory, deletes original files from storage and encrypts them within memory. In this case, DIM retains a backup copy of the targeted file and can recover it even the file is deleted by fileless ransomware.

\section{Digital IMMUNiTy}

This section describes our proposed DIM. The primary role of DIM is to prevent ransomware encrypt DIM-protected files. DIM is file-focused and its objective is twofold:

- detect when DIM-protected files are being encrypted,

- prevent encryption.

\section{A. Encryption Detection - Shannon Entropy Approach}

To this end, DIM must have an ability to distinguish encrypted files from unencrypted ones. Unencrypted files include text files (such as PDF, Microsoft Word and Latex documents) and multimedia files (such as pictures, music and movies/video). As a natural distinguisher, we propose the entropy concept as formulated by Shannon [7]. Given a random variable $X$ determined for $n$ events $x_{1}, \ldots, x_{n}$, where each event occurs with probability $P\left(x_{i}\right) ; i=1, \ldots, n$. Entropy is defined as

$$
H(X)=-\sum_{i=1}^{n} P\left(x_{i}\right) \log _{2} P\left(x_{i}\right) .
$$

We assume that events of our random variable $X$ are characters of the Unicode standard as well as non-printable Unicode characters. Entropy allows us to characterise how random is the content of a file. High entropy means that the content is random, while a low entropy indicates highly redundant (correlated) content. To calculate an entropy $H(f)$ of a file $f=\{x\}_{1}^{N}$ with $N$ characters from $X$, it is enough to compute the number of occurrences $\ell_{x}$ for each character $x \in f$ as

$$
H(f)=-\sum_{x \in f} \frac{\ell_{x}}{N} \log _{2} \frac{\ell_{x}}{N},
$$

where $\frac{\ell_{x}}{N}$ indicates the probability that the character $x$ occurs in the file $f$. For example, consider a file $f=\{$ aaaaaaaaa $\}$. This file consists of nine occurrences of "a" so $\ell_{a}=9$ and as $N=9$, then $\frac{\ell_{a}}{N}=1$ and $H(f)=0$. Consider another file $f^{\prime}=\{$ aeaieou! $\}$. A count of occurrences gives $\ell_{a}=2$, $\ell_{e}=2, \ell_{i}=1, \ell_{o}=1, \ell_{u}=1$ and $\ell_{!}=1$. It is easy to check that $H(f)=2.5$.

We assume that each type of unencrypted file is treated as a source of symbols/characters. We further suppose that its specific probability distribution can characterise the source program. Thus, for a long enough file $f$ generated by a PDF source (for instance), the probability of a symbol $x$ can be closely approximated by the occurrence $\ell_{x}$ of $x$ or

$$
P(x) \approx \frac{\ell_{x}}{N},
$$

where $N$ is the number of characters in the file $f$. In other words, $H(f)_{P D F}=H_{P D F}$, where $H(f)_{P D F}$ is the entropy of $f$ generated by PDF while $H_{P D F}$ is the entropy of the PDF source. Note that this approximation does not work if a file $f$ is "short". Since we assume that sources of unencrypted files generate byte characters, the maximum entropy $H_{\max }$ of a file is equal to 8 bits. This happens if source characters occur with the uniform probability distribution.

\section{B. Entropy of Encrypted Files}

It is expected that (long enough) encrypted files should have an entropy close to $H_{\max }$. It is known that cryptographically strong encryption produces cryptograms that are indistinguishable from truly random ones. To confirm this hypothesis, we performed experiments using a few file types. First, we compute the entropy of a sample of a given file type. We then repeat the entropy computation for the sample after being encrypted by three different open-source encryption programs, namely, AESCrypt, GnuPG, and Challenger. Table I shows the results. We can confirm with a high probability that a file $f$ is encrypted if its entropy is $7.99<H(f) \leq 8$. However, it must be noted that compressed files may also achieve entropy close to $H_{\max }$.

TABLE I: Entropy of encrypted and un-encrypted files

\begin{tabular}{lcccc}
\hline File Format & Unencrypted & Challenger & GnuPG & AESCrypt \\
\hline .pptx & 7.90786434941 & 7.99993409023 & 7.99992547868 & 7.99992691156 \\
.bmp & 6.38231379202 & 7.99994411725 & 7.99969271027 & 7.99994075204 \\
.jpg & 7.96841466702 & 7.99969969991 & 7.99972370710 & 7.99964407683 \\
.docx & 7.82881687843 & 7.99564129666 & 7.99531941975 & 7.99383985158 \\
.pdf & 7.85009879424 & 7.99998526860 & 7.99997917863 & 7.99998576992 \\
.xlsx & 7.93289273016 & 7.99846117766 & 7.99844636333 & 7.99869412812 \\
.png & 7.98653176951 & 7.99983209472 & 7.99978322342 & 7.99982432301 \\
.tex & 4.81546551317 & 7.99832884790 & 7.99416858853 & 7.99793348066 \\
.sql & 5.14310355930 & 7.99991774345 & 7.99949416462 & 7.99992064505 \\
\hline
\end{tabular}

The above considerations lead us to the following conclusions:

- Entropy can be used to characterise redundancy in files generated by a particular application, such as PDF, Microsoft Word and PowerPoint. The entropy of plain/unencrypted files is lower than $H_{7.99}$.

- Encrypted files exhibit a uniform probability distribution of characters, with entropy close to $H_{\max }$. An important exception is compressed files that also have high entropy values.

\section{Measuring Entropy in Practice}

Calculations of file entropy normally require an entire file to be fetched from HD to RAM. This is computationally 
expensive and time-consuming, especially for large files. Time and computational heavy detection processes are proved to be inefficient in dealing with ransomware attacks. This is due to the fact that once ransomware initiates attacks, it swiftly encrypts all target files. A practical solution is to find a 'minimum threshold length of bytes' where a portion of the target files can be used to approximate its entropy. The approximation should produce a value that is close enough to the real file entropy. This allows DIM to make a quick decision about whether or not a file is encrypted. To this end, we used 436 PDF documents containing historical Federal Reserve projections of the US economy, known as Greenbook projections (or Board of Governors Datasets). These PDFs are made up of text with tables and graphs interspersed throughout. Our experiments show that 25,000 bytes can be set as a minimum threshold length that allows evaluating file entropy with acceptable confidence - see Table II.

\section{Entropy of Compressed Files}

a) JPEG files: Compressed files naturally tend to have high entropy. For instance, the widely-used JPEG (lossy) compression removes the redundancy of characters in image files. For our experiments, we used the INRIA Holidays JPG dataset [8]. The dataset contains 812 JPG images and takes $1.1 \mathrm{~GB}$ of storage. We calculate entropy for every file in the dataset. Out of 812 results, the highest entropy is 7.95245506 (calculated from the first 25,000 bytes of the file). Figure 1 illustrates the results of our experiment. Note that, although the entropy values of JPG files are high, they are not as high as the entropy values of encrypted files. This noticeable redundancy is due to the fact that the probabilities of some characters do not follow a uniform distribution. For instance, the Null (or $00)$ character occurs with the highest probability in a majority of JPG files. In other JPG files, the $n b s p$ (or 255) character has the highest frequency.

b) TIFF files: TIFF applications use adaptive dictionary algorithms such as the LZW compression. We perform experiments to find statistical properties of TIFF files. We use a sample of 87 TIFF images taken from a medical database, i.e., OME-TIFF dataset. It turns out that the highest file entropy is 5.20967171, which is significantly lower than $H_{\max }$ of 8 . Consequently, we observe a non-uniform probability distribution of file characters. For example, the $N u l l$ character occurs with the highest probability in TIFF files.

High-performance compression algorithms such as those used in TAR and ZIP applications reduce character redundancy so well that file entropy becomes very close to $H_{\max }$. This essentially makes distinguishing compressed files from ransomware-encrypted files a challenging task, especially if entropy is the only characteristic used. To mitigate this difficulty, user applications and DIM can negotiate an acceptable quality of compression. This could be done by skewing byte probability distribution that introduces $\varepsilon$ redundancy. As a result, the file entropy becomes $H(f)-\varepsilon$, where $H(f)$ is the entropy of the compressed file. The redundancy $\varepsilon$ should be small so that the compression quality does not suffer. At the same time, $\varepsilon$ should be big enough so that DIM is able to tell apart compressed files from encrypted ones.

\section{E. Distribution of Symbols in Encrypted Files}

Characters in encrypted files behave as random ones, and consequently, it is impossible to extract any sensitive information from them. In fact, any two neighbouring characters should be looked at as independent random variables $X$ and $Y$, where $X$ does not reveal any information about $Y$ and vice versa. As the result, characters in encrypted files follow a uniform probability distribution. To verify whether this conclusion is true for ransomware encryption, we select six wild ransomware variants and encrypt a collection of the three datasets, i.e., Greenbook projections, INRIA Holidays and OME-TIFF. We compute standard deviations of characters for the first 25 kilobytes (KB) of each encrypted file. Figure 2 illustrates the distribution of symbols in both unencrypted and ransomware encrypted files. This confirms the fact that characters in encrypted files are uniformly distributed. Figure $2 \mathrm{~h}$ shows standard deviations of all encrypted files combined together (the total of 8010 files). Additionally, Table III depicts the confidence intervals for both encrypted and unencrypted files calculated for the first $25 \mathrm{~KB}$ of the 8010 encrypted and 1335 unencrypted files, i.e., $99 \%$ to $80 \%$.

a) Ransomware with Low Entropy: Today, digital devices have more than one multi-core CPUs with simultaneous multi-threading or hyper-threading technology. These advancements in microprocessor technology deliver huge performance benefits for day-to-day business operations, allow parallel executions and improve system utilisation. Malware developers also take advantage of the progress in microprocessor technology. They use advanced CPUs and parallelise specific tasks to accelerate their activities. New generation ransomware achieves higher throughput and a lower latency by using parallel threads. It is able to concurrently fetch data from HD and encrypt them without waiting for other threads to finish. A good example of such ransomware is Dharma (also known as CrySIS) that has been present since 2016. There are more than a hundred different Dharma versions. Based on our analysis, this multi-threaded ransomware encrypts eight files at a time. It also writes $256 \mathrm{~KB}$ of the null character at the beginning of image files (such as JPG and TIFF), we suspect that the intention of that unusual file modification was to prevent encryption detection by entropy checking. The addition of null bytes lowers file entropy values. To mitigate this, we apply a sliding window while reading the (initial) $25 \mathrm{~KB}$ of a file to detect encryption. The sliding window technique is described in the following section.

\section{F. Context Triggered Piecewise Hashing}

Similarity-preserving hashing (a.k.a. fuzzy hashing) was used for malware analysis [9]. For the purpose of this study, we propose to use fuzzy hashing for detecting buffers with malicious contents. Given a collection of files $F=$ $\left\{f_{1}, f_{2}, \ldots, f_{m}\right\}$, each file $f_{m}$ to be read requires a finite set of buffers $\left\{\left|b_{1}, b_{2} \ldots, b_{n}\right|\right\}$, where $b_{n}$ has the size of 4096 bytes. Let $f_{\text {hash }}\left(f_{m}\left(b_{n}\right)\right)$ be a hashing signature (digest) of the $b_{n}$ buffer contents of $f_{m}$. Algorithm 1 calculates similaritypreserving hash signatures for all files from the collection $F$ stored on HD. This helps us to (1) detect files that have correct 
TABLE II: Entropy approximation of 15,000 bytes (left) vs. 25,000 bytes (right) for Greenbook PDF Dataset encrypted by the named ransomware. Bold values are used to determine the threshold value of 7.99 - only a sample of files shown here.

\begin{tabular}{|c|c|c|c|c|c|c|c|c|c|c|c|c|c|c|c|}
\hline \multirow{2}{*}{ File Name } & \multirow{2}{*}{$\begin{array}{l}\text { Entropy of } \\
\text { the entire file }\end{array}$} & \multicolumn{7}{|c|}{ The first 15000 bytes of the file are read and the entropy is computed. } & \multicolumn{7}{|c|}{ The first 25000 bytes of the file are read and the entropy is computed. } \\
\hline & & GandCrab & locked & OFFWHITE & az3zg & avdn & eswasted & WNCRY & GandCrab & locked & OFFWHITE & az3zg & avdn & eswasted & WNCRY \\
\hline & & 7.8 & 7.98718 & 7. & 7 & 34 & 7. & 7. & 7.99 & & & & & & \\
\hline & & & & & & & & & & & & & & & \\
\hline & & & & & & & & & & & & & & & \\
\hline & & & & & & & & & & & & & & & \\
\hline & & & 7.988 & & & & & & & & & & & & 4339 \\
\hline-196 & 7.718 & $\begin{array}{r}7.987 \\
7.987 \\
\mathrm{C}\end{array}$ & 7.989 & 7.9889 & 7.987 & 7.987567688 & 7.989 & 7.987 & 7.999 & 01 & $\begin{array}{c}7.9932 \\
7.992\end{array}$ & $\begin{array}{l}7.99315747 \\
7.9937553\end{array}$ & 597 & 7.992335577 & 13543 \\
\hline $966-06-06-28$ & 7.786 & 7.986 & 7.9884 & 7.988 & 7.9 & 7.98 & 7.9887 & 7.98 & 7.993 & 7.99 & 7.99 & 7.993 & 433 & 476 & 1798 \\
\hline & & & & & & & & & & & & & & & \\
\hline $66-08-23$ & & 14 & 4 & 7.98884781 & 636617 & 7.9893225 & 725 & 7.9 & 28 & $\mathbf{7 . 9 9 3 5 6 6 7 9 5}$ & 67 & 53 & 44 & 7.9 & 7.99 \\
\hline
\end{tabular}

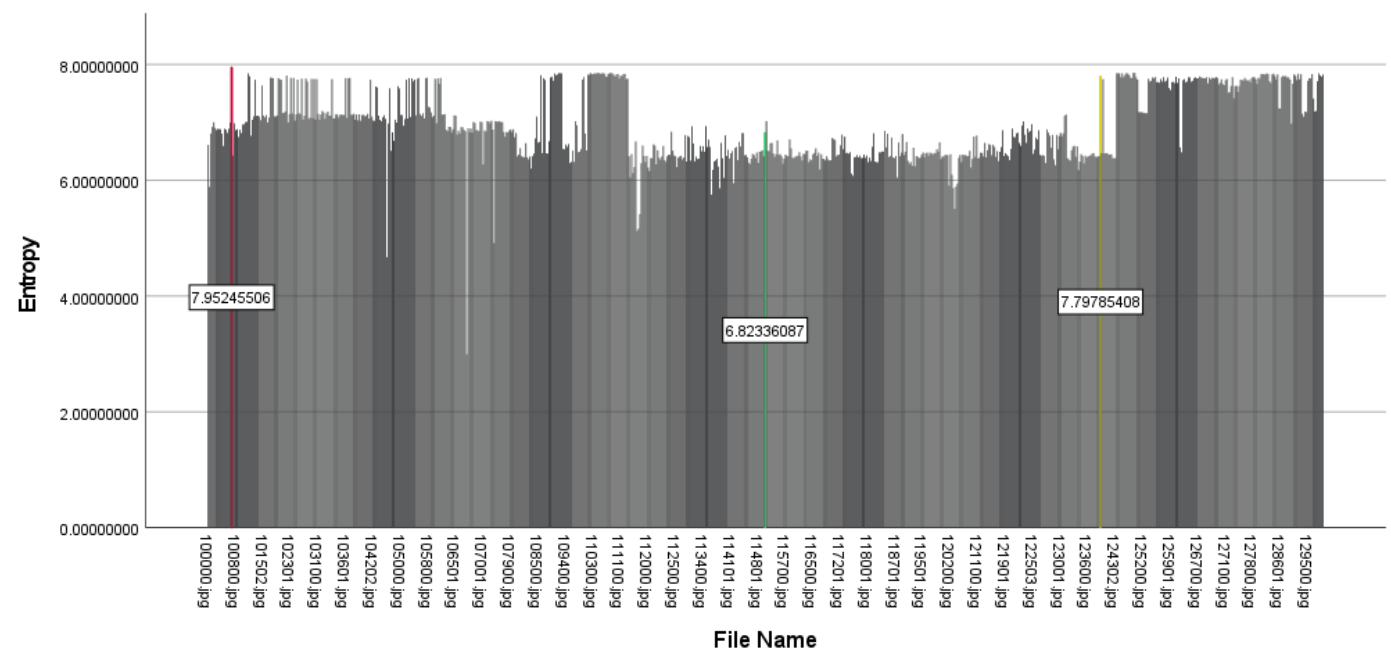

Fig. 1: Entropy of 812 JPG files.

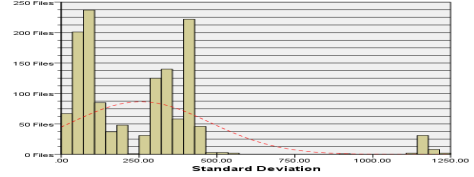

(a) Unencrypted files

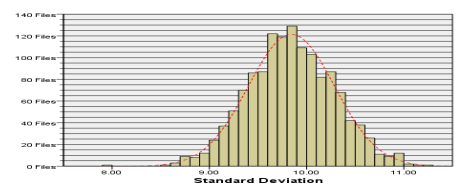

(d) GandCrab

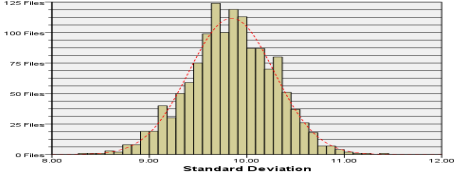

(b) AVDN

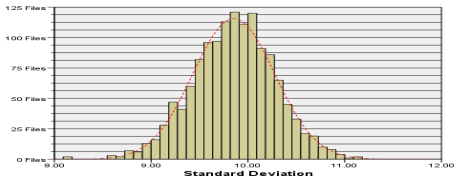

(e) OFFWHITE

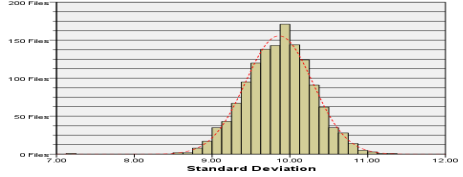

(c) Eswasted

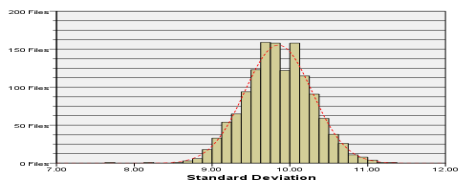

(f) Sodinokibi

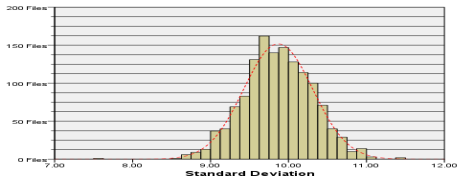

(g) Wannacry

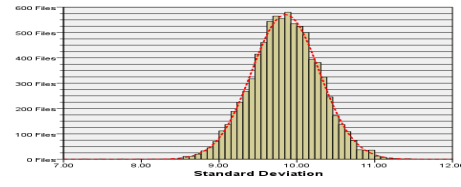

(h) 8106 encrypted files

Fig. 2: Standard Deviation $(\sigma)$ of PDF, JPG, TIFF: computed from the first $25 \mathrm{~KB}$ of each file. Sub-Figure 2a shows symbols appear loosely distributed throughout unencrypted files, whereas in encrypted files, symbols are fairly distributed.

similarity-preserving hash signatures before writing them to HD; (2) identify padding data added by ransomware in order to reduce file entropy; (3) make it difficult for ransomware to guess, which buffer hash signatures have been chosen by DIM to identify encrypted contents; (4) check if ransomware has deleted and rewritten all buffers of a file as in this case, the original file is damaged and no ransom should be paid; (5) update buffer fuzzy signatures after each legitimate file write operation; (6) tell apart existing files from new ones even if the new ones have high entropy (zip or encrypted file). 


\begin{tabular}{|l|c|c|c|c|c|c|}
\hline & \multicolumn{3}{|c|}{ Encrypted files } & \multicolumn{3}{c|}{ Unencrypted files } \\
\cline { 2 - 7 } & Lower bound & Mean & Upper bound & Lower bound & Mean & Upper bound \\
\hline $\begin{array}{l}\text { Confidence } \\
\text { Interval 99\% }\end{array}$ & 96.02748789 & 97.65625 & 99.31896542 & 35.4255017 & 97.65625 & 159.8869983 \\
\hline $\begin{array}{l}\text { Confidence } \\
\text { Interval 95\% }\end{array}$ & 96.38530503 & 97.65625 & 98.92719497 & 50.43465809 & 97.65625 & 144.8778419 \\
\hline $\begin{array}{l}\text { Confidence } \\
\text { Interval 90\% }\end{array}$ & 96.590831 & 97.65625 & 98.721669 & 58.07091603 & 97.65625 & 137.241584 \\
\hline $\begin{array}{l}\text { Confidence } \\
\text { Interval 85\% }\end{array}$ & 96.72440466 & 97.65625 & 98.58809534 & 63.03380651 & 97.65625 & 132.2786935 \\
\hline $\begin{array}{l}\text { Confidence } \\
\text { Interval 80\% }\end{array}$ & 96.8330564 & 97.65625 & 98.4794436 & 66.84643556 & 97.65625 & 128.4660644 \\
\hline
\end{tabular}

TABLE III: Average Confidence Interval of the first 25000 Bytes of 8010 encrypted and 1335 unencrypted files. The data set is encrypted by six different ransomware families.

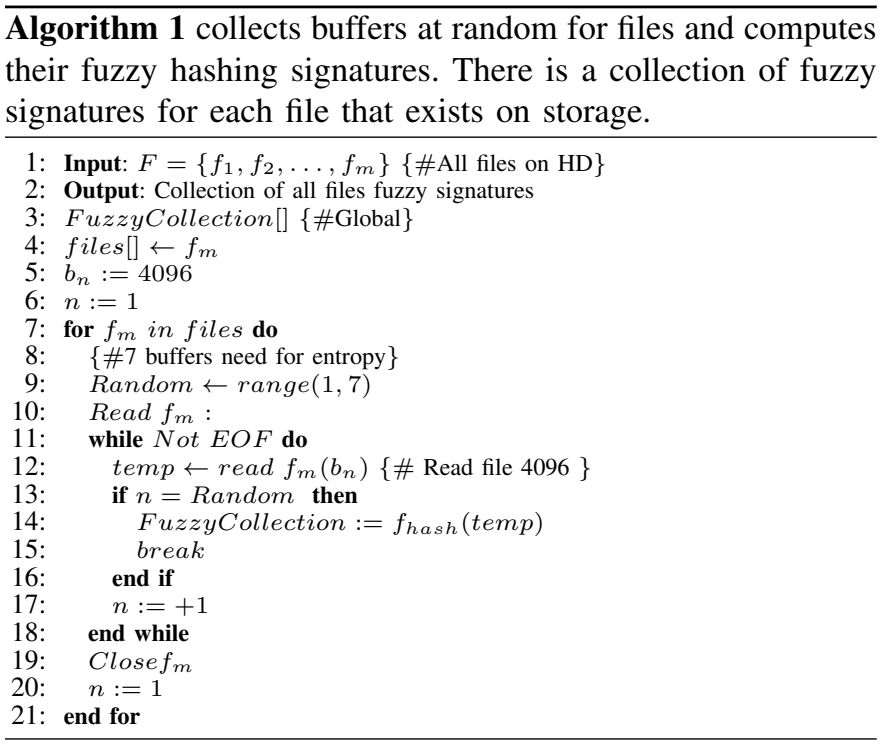

\section{DIM AlgorithmS AND Modules}

Our ultimate goal is to implement DIM as a storage firmware. The following setup is used as a proof of concept. The implementation setup consists of a Dell Desktop with 16 GB of RAM and an i7-4770 CPU @ 3.40GHz, 4 Cores, 8 Logical Processors. Two virtual machines run on a VMware hypervisor configured with $4 \mathrm{~GB}$ of RAM and 4 processor cores for each virtual machine. We use Ubuntu 20.04 Desktop and Windows 10 Education $\mathrm{N}$ operating systems on virtual machines. We have configured and installed Windows 10 on the first virtual machine (VM) as the victim's computer. Because Linux and Mac operating systems have a smaller market share, most ransomware is developed for the Windows kernel architecture. On the second VM, Ubuntu shares files with users on the network using the Samba protocol as the network storage protected by DIM. A shared folder located on the Ubuntu VM contains 1335 files (pdf, jpg, and tiff). A module of the Window VM interacts with a user-space file system (FUSE) [10] installed on the Ubuntu VM.

The FUSE file system in the user-space is the most widely used user-space file system framework. According to modest estimates, at least 100 different FUSE-based file systems are readily available on the Web. FUSE consists of a kernel part and a user-level daemon. The kernel part is implemented as a Linux kernel module. After loading, it registers a FUSE file system driver with the Linux virtual file system (VFS). The FUSE driver acts as a proxy for various specific file systems. They are implemented by different user-level daemons as illustrated in Figure 3 FUSE is implemented for Windows systems and is called WinFsb. When a user application performs operations on a mounted FUSE file system, the VFS routes the operation to a FUSE kernel driver. The driver allocates a FUSE request structure and puts it in a FUSE queue. At this point, a process that has submitted the operation is usually put on hold. The FUSE user-level daemon then picks up the request from the kernel queue by reading from /dev/fuse and processes the request $[10]$.

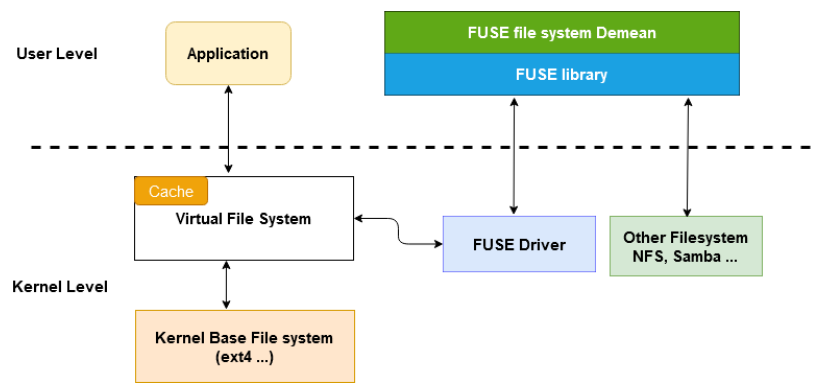

Fig. 3: FUSE architecture creates a bridge between Virtual File System and FUSE driver.

\section{A. DIM Pass-through Module for Encryption Detection}

We have developed a pass-through module that interacts with the FUSE file system. A pass-through module intercepts and inspects the read/write buffers of every file. The module is equipped with all the necessary permissions to manipulate files. This includes access, chmod, open, create, read and write. So it is a fully functional file system. There are three main directories $\mathbf{A} \Leftrightarrow \mathbf{B} \Leftrightarrow \mathbf{C}$ that interact with the passthrough module. The directory $\mathbf{A}$ contains all the files and is not directly accessible to users. $\mathbf{B}$ is a shared mounting point (user-level daemon) that is configured with the Samba protocol and is shared with users over the network. $\mathbf{C}$ is located in the user environment. It is the only directory that users can access. Note that both $\mathbf{A}$ and $\mathbf{B}$ are located on the Ubuntu VM but $\mathbf{C}$ is located on the Windows VM, which is the user machine. When a process (called by either a user or ransomware) opens or writes a file, the module inspects the file's write buffers.

The pass-through module inspects all write buffers of files coming from the directory $\mathbf{C}$. It checks whether a buffer contents is encrypted before it writes the buffer contents to HD. According to our analysis, we need at least $25 \mathrm{~KB}$ of the file to compute a file entropy. The module reads seven buffers (4096 bytes each) to identify encrypted content and calculates a file entropy, standard deviation and confidence interval. Assume that ransomware reads a $100 \mathrm{~KB}$ file, encrypts its contents, and stores it in the memory, i.e., buffer cache. Next, the system uses a default block size and writes the encrypted bytes to HD. In this case, the block size is $4 \mathrm{~KB}$. Let $\Omega=\left\{b_{1}, b_{2}, \ldots, b_{n}\right\}$ be a collection of $n$ buffers. Encryption detection procedure is shown in Algorithm 2 .

\section{B. DIM Encoding Expansion Module}

Compression is a special code that assigns short bit encodings to more probable symbols. On the other hand, less probable symbols are encoded into longer bit encodings [11]. There 


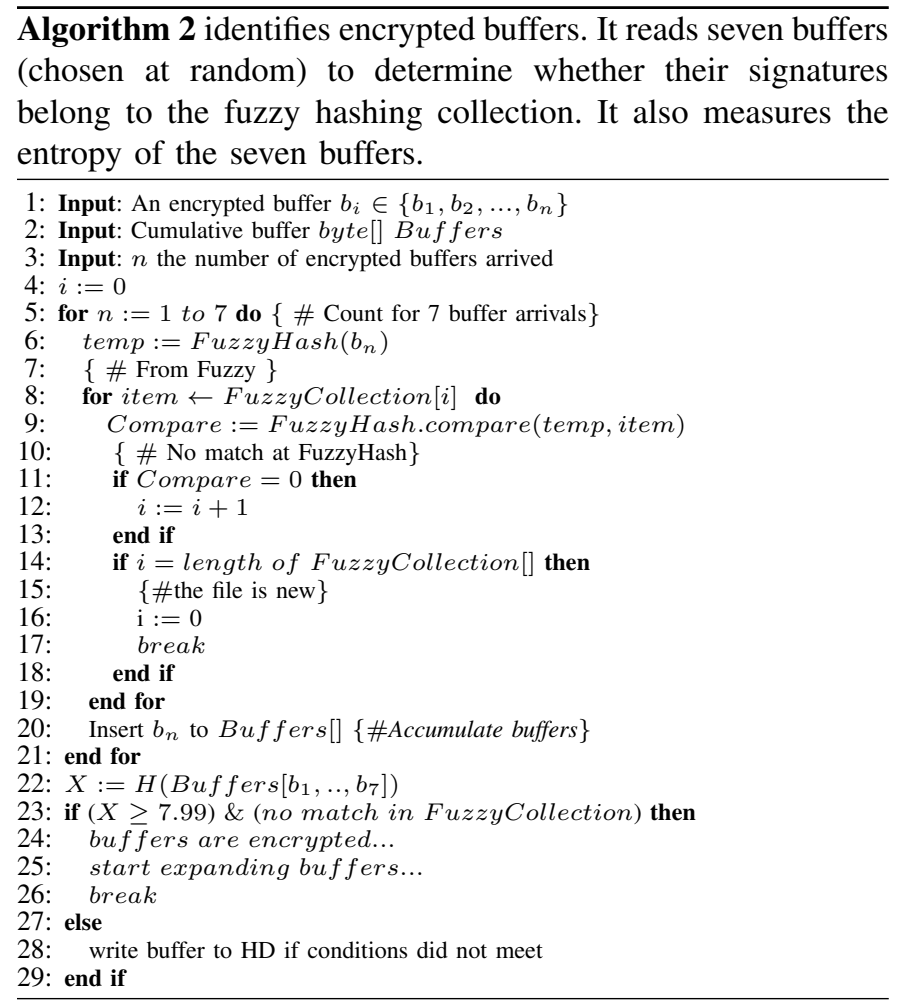

is a rich literature that covers coding schemes for lossless compression, including Huffman, arithmetic, the Lempel-Ziv family (see [11]) and the asymmetric numeral system (ANS) [12] outperformed previous methods by being up to 30 times faster.

Compression is an indispensable tool that shortens transmission time, saves storage space and speeds up encryption. Interestingly enough, if the occurrences of symbols in a file do not follow the prescribed symbol statistics, then compression expands the file (instead of compressing). This normally undesired effect can be used as a useful defence against ransomware. To be exact, assume that DIM is asked by ransomware to store an encrypted file on HD. Then DIM applies a compression algorithm using non-uniform symbol statistics, e.g., symbol occurrences coming from or similar to the original file before the ransomware encrypts it. Such DIM compression expands the file and keeps the ransomware encryption engine busy. A size of expansion can be controlled by changing symbol probabilities. In general, expansion size grows when more frequent symbols are assigned very low probabilities. In theory, expansion can reach sizes of GBs (or even TBs). Consequently, DIM slows down the ransomware encryption engine. This delay allows DIM to set an alarm for a system/human intervention. It is theoretically possible to increase symbol probabilities to altogether disable ransomware functionality through heavy I/O load or insufficient storage in DIM controlled volume.

The main task of an encoding expansion module is to engage a ransomware encryption engine by feeding it with an ever-expanding bitstream. For this purpose, we developed a simple algorithm that takes a stream of symbols with uniform distribution (the case of an encrypted file) and produces a very long bitstream. Assume that ransomware requests to write an encrypted file to a victim's HD. Before progressing any further, we need to introduce some notations. Let $C=\Delta=$ $\left\{c_{1}, c_{2}, \ldots, c_{m}\right\}$ be a collection of characters (Unicode). For a sequence $S=\left(s_{1}, \ldots, s_{n}\right)$, where $s_{i} \in \Delta,|S|_{c_{i}}$ gives the number of occurrences of $c_{i}$ in the sequence $S$. The probability of occurrence of $c_{i}$ in $S$ is $P\left(c_{i}\right)=\frac{|S|_{c_{i}}}{n}$.

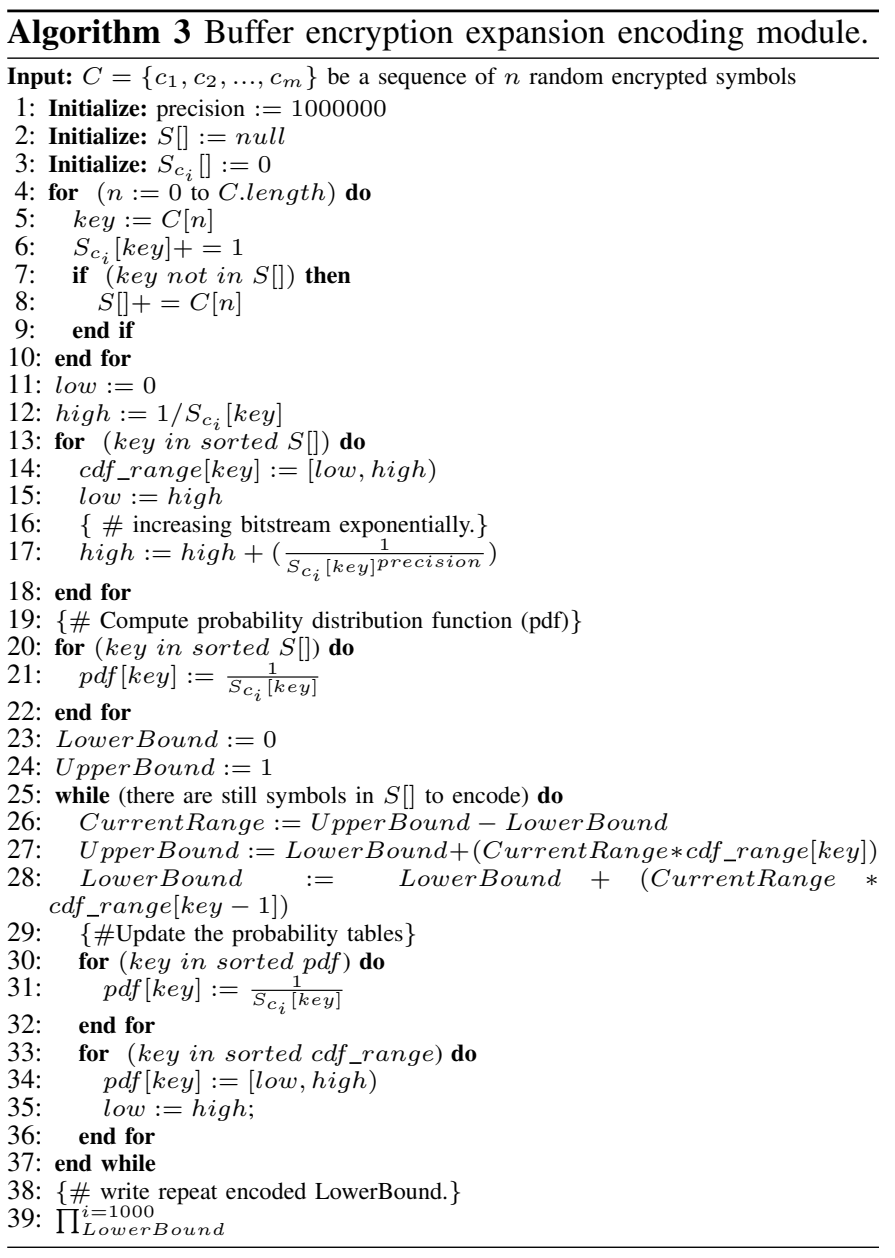

Algorithm 3 works with an interval $[0,1)$ of real numbers. As a set $C$ grows, the interval needed to represent it shrinks, but the number of bits needed to specify the interval grows. Symbols $\left\{c_{1}, c_{2}, \ldots, c_{m}\right\}$ of the set of encrypted symbols reduce the size of the interval in accordance with the symbol probabilities. The algorithm should allow error-free decoding in the case when the expansion encoding module has encoded unencrypted symbols. To achieve this, we increased the precision of the symbol distribution range table.

Let us illustrate the algorithm on two examples. Given a set of random unencrypted symbols $\{a, e, i, n, t\}$. Arithmetic encoding is exemplified in Table IV Each symbol $s$ is assigned a unique interval, whose length is proportional to the symbol probability. Assume that our encoding expansion module receives the sequence $(e, a, i)$ of symbols. Seeing the first symbol $e$, the module narrows the initial interval $[0,1)$ to $[0.2,0.5)$. For the second symbol $a$, it takes the current interval $[0.2,0.5)$ and shortens it proportionally to $[0.2,0.26)$ The lower interval bound is $p_{s_{1(l o w)}}$, while the upper interval 
bound is $p_{s_{1_{(l o w)}}}+p_{s_{0}} p_{s_{1}}$. For the last symbol $i$, the current interval $[0.2,0.26)$ is proportionally narrowed to $[0.23,0.236)$. The lower interval bound is $p_{s_{1}(\text { low })}+p_{s_{1}} p_{s_{2}}$, while the upper interval bound is $\left(p_{s_{1_{(l o w)}}}+p_{s_{1}} \cdot p_{s_{2}}\right)+p_{0} p_{1} p_{2}$. Finally, the module stores the lower bound 0.23 as an encoding of $(e, a, i)$. Now consider a sequence of symbols $\{x, y, z, k, !$, Space $\}$.

TABLE IV: Example of fixed normal symbols distribution range model.

\begin{tabular}{cllll}
\hline Symbols & \multicolumn{2}{c}{ Probability } & \multicolumn{2}{c}{ Range } \\
\hline $\mathrm{S}$ & $p_{s_{n}}$ & $p_{s_{(\text {low })}}$ & $p_{s_{(\text {high })}}$ \\
\hline $\boldsymbol{a}$ & $p_{s_{0}} \leftarrow$ & 0.2 & {$[0.0$,} & $0.2)$ \\
$\boldsymbol{e}$ & $p_{s_{1}} \leftarrow$ & 0.3 & {$[0.2$,} & $0.5)$ \\
$\boldsymbol{i}$ & $p_{s_{2}} \leftarrow$ & 0.1 & {$[0.5$,} & $0.6)$ \\
$\boldsymbol{n}$ & $p_{s_{3}} \leftarrow$ & 0.3 & {$[0.6$,} & $0.9)$ \\
$\boldsymbol{t}$ & $p_{s_{4}} \leftarrow$ & 0.1 & {$[0.9$,} & $1.0)$ \\
\hline
\end{tabular}

Assume that the probability distribution of the symbols is given in Table $\mathrm{V}$. Figure 4 illustrates the probability density function range table with a precision of 15 (see Algorithm 4). We also present the output of expansion of encrypted symbols $S=\{x, y, z, k, !$, Space $\}$, i.e., 6 bytes, with 500 precision which increases the compression output to 672 bytes (see Figure 5). Larger precision can increase the compression output to arbitrarily large file sizes of a GB order. One million precision has been tested. It expands a 5 byte file to a 10GB one before system crashes - a desired event when a ransomware attack commences. In theory, it is possible to get expansion of a short file into much larger one of a TB order.

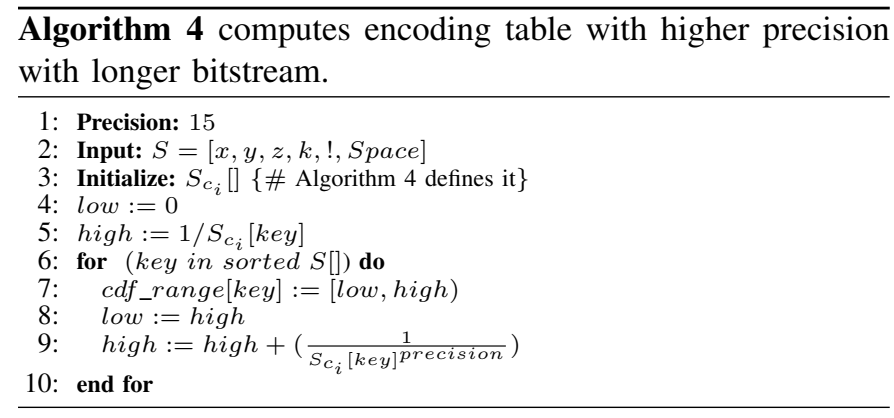

TABLE V: Probability density function range table for each symbol with equal distribution probability $\left(p_{0}, \cdots, p_{5}=\right.$ 0.166667).

\begin{tabular}{cccc} 
Symbols & Probability & \multicolumn{2}{c}{ Range $\leftarrow 1 /|S|$} \\
\hline $\mathbf{S}$ & $p_{s}$ & $p_{s_{\text {low }}}$ & $p_{\text {s }_{\text {high }}}$ \\
\hline Space & $p_{0}$ & {$[0.000000$,} & $0.166667)$ \\
$!$ & $p_{1}$ & {$[0.166667$,} & $0.333334)$ \\
$\boldsymbol{k}$ & $p_{2}$ & {$[0.333334$,} & $0.500001)$ \\
$\boldsymbol{x}$ & $p_{3}$ & {$[0.500001$,} & $0.666668)$ \\
$\boldsymbol{y}$ & $p_{4}$ & {$[0.666668$,} & $0.833335)$ \\
$\boldsymbol{z}$ & $p_{5}$ & {$[0.833335$,} & $1.000000)$ \\
\hline
\end{tabular}

\section{Recovery of Corrupted Files}

Newly developed ransomware overwrites original files immediately. It reads the first few files, deletes the original ones

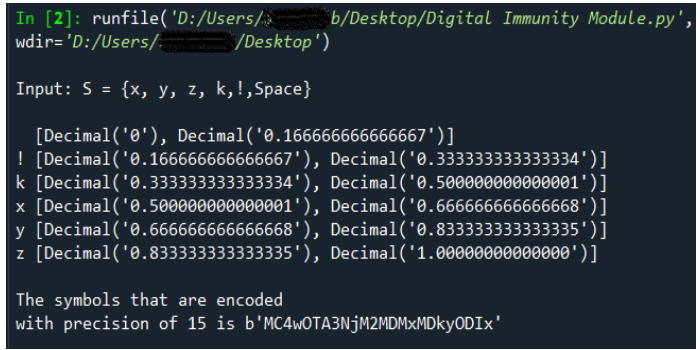

Fig. 4: The probability density function range table with precision of 15 .

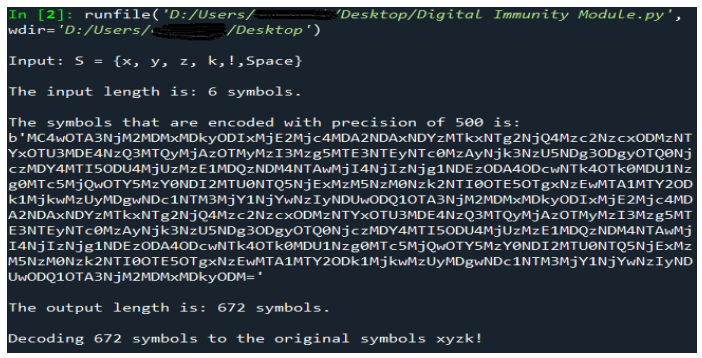

Fig. 5: Manipulating the probability density function range table with precision of 500 which increases 6 symbols inputs to 672 symbols of the outputs.

and writes encrypted files from memory to storage. As a result, before DIM detects maliciously encrypted files, ransomware has already altered a few files. DIM includes a module that keeps every file being opened/read in a temporary directory that is not accessible to user processes. The module reads buffers and gathers file statistics to ensure a recovery path. Once DIM has concluded that the buffer contents is not encrypted, it deletes the temporary directory. Otherwise (if the contents is encrypted), DIM keeps the directory for file recovery purposes. Because ransomware can only access and encrypt a limited number of files at once, the maintenance of the temporary directory neither overburdens the HD storage nor adds a significant OS computational overhead. The rescue module also adds another layer of protection against file encryption that may be deployed by future ransomware. In other words, even when ransomware employs complex encryption and compression techniques to avoid detection by statistical analysis, the rescue module ensures that no file is lost. So far, we have not come across a ransomware variant that employs an unusual encryption technique that our DIM cannot deal with because DIM purely focus on the protected file rather than the ransomware code.

\section{Evaluation And Results}

We have gathered a large collection of ransomware from different malware repositories. It includes well-known representatives such as VirusShare, MalwareBazaar, and Kaggle. We collected 39 ransomware families and their variants illustrated in Tables VII and VIII. They can be executed without relying on $C \& C$ communication. We evaluated the effectiveness of DIM's file encryption prevention functionality against our file dataset, which includes 1335 files of pdf, jpg, and tiff formats. 
We placed the file dataset in a shared drive folder over the network and executed each ransomware variant for the dataset.

DIM reads all file buffers using sliding windows and computes entropy, standard deviation, and confidence interval values on the fly. As discussed earlier, this file slicing approach reduces the computational cost of detecting encrypted files. For most ransomware variants, the first window of seven buffers is sufficient for detecting encryption. However, some ransomware variants attempt to lower entropy by inserting redundant characters. For instance, Dharma injects $250 \mathrm{~KB}$ of null characters at the beginning of a file. Note that adding $250 \mathrm{~KB}$ of null characters may also deceive anti-malware software that checks file headers. To deal with this deception, DIM employs sliding windows. In the case of Dharma, DIM has to read nine windows (nine sets of seven buffers) and only from the 8-th window of seven buffers, i.e., buffers 64-70 (see Figure 6, DIM is able to detect that the buffer contents is encrypted. This triggers DIM to start expanding the buffer contents.

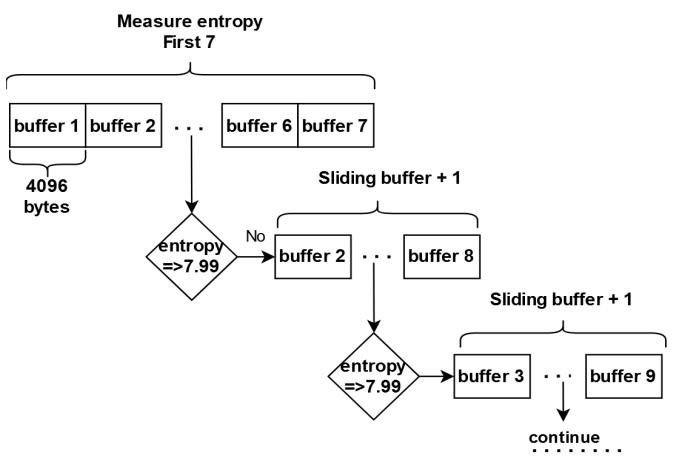

Fig. 6: DIM uses sliding windows to deal with ransomware, which may insert characters to reduce entropy.

DIM's file expansion module successfully engages ransomware by continuously expanding files. For example, DIM can expand a small file of $4 \mathrm{~KB}$ to $10 \mathrm{~GB}$ or larger sizes. Based on our experiments and analysis, all collected ransomware variants stop file encryption once the files under attack are expanded. DIM's file expansion module offers a specific advantage of file recovery in case of a false positive detection. DIM can recover encrypted files to their original files. We have established that DIM exhibits zero false negative detection through our extensive experiments, including the most recent ransomware attacks.

The results of computed statistics during ransomware executions indicate that the entropy of encrypted data reaches above the optimum threshold value of 7.99 for almost all files. Similarly, both standard deviation (STD) and confidence interval (CI) values also attain optimum threshold values. Some ransomware variants from our experimental collection, however, do not attack shared drive folders. Our experiments have confirmed that DIM is effective in protecting files on shared drives against ransomware attacks. We compared DIM's CPU, RAM and DISK I/O resource usage during ransomware attacks and idle operations remotely. During ransomware attacks, and in the absence of DIM, the average total CPU usage (all cores) in Linux OS was $91 \%$. On average, ransomware took
3 minutes and 32 seconds to encrypt the entire file dataset. During a ransomware attack, DIM consumed at most $81 \%$ of the total CPU usage (all cores) available. The results testify that the operation of DIM does not add computational overhead and shortens the computational costs imposed by ransomware. Although due to DIM's load demand, disk I/O continues to increase while the file under attack is expanded. This is essentially a shift in the usage of disk $\mathrm{I} / \mathrm{O}$ resources from ransomware to DIM.

\section{DIM VERSUS BINTROPY MODELS}

We use statistical techniques to analyse contents of encrypted buffers. A gathered statistics is used to detect encryption and to defend files against ransomware activities. Our entropy-based approach differs significantly from the bintropy-based approach described in [13]. Bintropy uses a statistical test suite based on discrete Fourier transform. It detects repetitive patterns that are close to each other. Bintropy aims to identify whether a binary file includes compressed or encrypted bytes. It calculates the amount of statistical variation in bytes for a data stream. It sums the frequency of each observed byte value $(00 \mathrm{~h}-\mathrm{FFh})$ that exists in fixed-length data blocks and then uses the entropy to compute entropy scores. Standard sections are included in a standard compilergenerated PE executable, i.e., .text, .rsrc, .reloc and .data file extensions. Many packing tools alter the original executable format by compressing and collapsing the standard section code and data into one or two new sections.

Bintropy calculates an entropy score for each section. It does not compute a score for the header section, which is unlikely to contain encrypted or compressed bytes. It also examines the entropy of the entire file, beginning with the first byte and ending with the last. It can thus compute the entropy of code or data hidden at the end of a file or between PE-defined sections (cavities). This is usually a place where a stealthy file-infecting code is typically hidden. It is difficult to determine whether an executable such as ransomware contains compressed or encrypted bytes. Bintropy can, in fact, generate both false positives and false negatives. False negatives can happen when large executable (greater than $500 \mathrm{~KB}$ ) contain a small number of encrypted or compressed blocks and a large number of valid blocks, lowering the executable entropy value. Sophisticated malware developers can use protective measures to try and hide the use of encrypted data or compression. They can, for example, use strong cryptography to pad encrypted bytes with redundant bytes. This is very much the same idea that is used by the Dharma ransomware. Furthermore, Bintropy cannot protect data from fileless ransomware or malware where no actual malicious binary file exists on the storage.

The presented DIM does not rely on ransomware executable files or malicious processes for detection. Instead, it relies on the file system write function and statistical analysis for what ransomware writes. Bintropy and other current ransomware detection programs can only detect malicious processes or executable files. DIM further offers a file encryption prevention mechanism, i.e., the encoding expansion module. 
TABLE VI: Important ransomware detection techniques.

\begin{tabular}{|c|c|}
\hline Detection techniques & Reference \\
\hline Behaviour Based & $\begin{array}{l}{[19} \\
{[22}\end{array}, \frac{20]}{2},[21]$, \\
\hline I/O Request Packer Monitoring & 15 \\
\hline Network Traffic Monitoring & $25,[26,, 27], 28], 29$ \\
\hline Storage Level & {$[16,[30],[17],[18$} \\
\hline API Call & [31] \\
\hline Others & [32, 33$], 34$ \\
\hline
\end{tabular}

\section{RELATED WORK}

Various approaches for detecting ransomware have been proposed [14]. They may observe the behaviour of processes, the flow of $I / O$ packets, network traffic or API calls. A significant body of work covers ransomware detection using machine learning models [15]. A few methods have been suggested to deal with ransomware activities at the storage level. ShieldFS [16] proposes to monitor low-level activities of a file system. It allows to update a set of adaptive models that characterize the behaviour of systems over time. The authors claim that ShieldFS can detect suspicious activities at run-time and can retrieve all original files. They developed a customized classifier and implemented a range of models. Based on our ransomware analysis, the distinction between normal user behaviour and malicious activity is limited to a number of applications. Authors suggest that shadowing original files from the earlier stage can eliminate false-negative and restore files from their copies. This is similar to a backup. New variants of ransomware find files even if they are on the network.

A ransomware detection mechanism on storage devices, especially on flash-based storage, is discussed in [17]. An access-pattern-based detector that can detect ransomware that overwrites an original file with encrypted file. The detector works in conjunction with a custom buffer management policy used by SSD to manage their read and write buffers. The buffer management policy keeps track of reads and writes to the same location to check for ransomware activity. The method fails when read-write buffers are relatively small. Baek et al. in [18] have proposed a new method to safeguard the system against ransomware inside NAND flash-based SSDs called SSD-Insider. They use block address, size, and type of an IO request to an SSD as features to capture the characteristics of ransomware. Moreover, they utilize a binary decision tree using the Iterative Dichotomiser 3 (ID3) algorithm to detect ransomware. Table VI classifies some of the most important ransomware detection techniques proposed in the literature.

\section{CONCLUSion, Limitation AND Future Work}

The paper proposes the digital immunity module (DIM), a novel approach for detecting encrypted buffers. DIM uses on-the-fly statistical analysis and prevents encryption using an inverse encoding scheme (e.g., arithmetic coding). The mechanism delays or can totally block ransomware encryption activities. We have collected representatives of 39 wild ransomware families and ran them against DIM to evaluate the effectiveness of DIM in real-world attack scenarios. A video demonstration of DIM in action against three ransomware variants as well as normal file system operations can be found in [35].

DIM's buffer expansion module forces ransomware to terminate at the very beginning of ransomware activities. The published ransomware detection methods are based on analyses of either file signatures, process behaviour, API calls or network traffics. Because these methods require access privileges to every kernel process, they can be quite intrusive, and they are crippled when it comes to fileless ransomware. Further, they do not allow to recover files lost during an initial execution of ransomware. In contrast, DIM guarantees an effective detection and prevention approach that significantly mitigates data loss and prevents malicious encryption during fileless ransomware attacks. A demo of DIM against fileless ransomware can be found in [36].

Having said this, we believe that none of the proposed models can be flawless. In the case of DIM, if a ransomware developer wants to successfully encrypt a victim's files without DIM detecting the attack, they must be able to reduce the entropy values of write buffers. According to our extensive ransomware analysis, no ransomware developer has employed unconventional entropy reduction methods that could bypass DIM. We have begun work on a proof-of-concept ransomware testing lab that may reduce encrypted file entropy. We will analyse the behaviour of such ransomware and improve the DIM accordingly, with a focus on protecting compressed files.

\section{REFERENCES}

[1] A. Young and M. Yung, "Cryptovirology: extortion-based security threats and countermeasures," in Security and Privacy, 1996. Proceedings., 1996 IEEE Symposium on, May 1996, pp. 129-140.

[2] A. Kharraz, W. Robertson, and E. Kirda, "Protecting against ransomware: A new line of research or restating classic ideas?" IEEE Security Privacy, vol. 16, no. 3, pp. 103-107, May 2018.

[3] H. Zhang, X. Xiao, F. Mercaldo, S. Ni, F. Martinelli, and A. K. Sangaiah, "Classification of ransomware families with machine learning based on n-gram of opcodes," Future Generation Computer Systems, vol. 90, pp. 211 - 221, 2019. [Online]. Available: http://www.sciencedirect.com/science/article/pii/S0167739X18307325

[4] Z. Wang, C. Liu, and X. Cui, "Evilmodel: Hiding malware inside of neural network models," CoRR, vol. abs/2107.08590, 2021. [Online]. Available: https://arxiv.org/abs/2107.08590

[5] R. Hasan, S. Myagmar, A. J. Lee, and W. Yurcik, "Toward a threat model for storage systems," in Proceedings of the 2005 ACM Workshop on Storage Security and Survivability, ser. StorageSS '05. New York, NY, USA: Association for Computing Machinery, 2005, p. 94-102. [Online]. Available: https://doi.org/10.1145/1103780.1103795

[6] R. Moussaileb, N. Cuppens, J.-L. Lanet, and H. L. Bouder, "A survey on windows-based ransomware taxonomy and detection mechanisms," ACM Comput. Surv., vol. 54, no. 6, Jul. 2021. [Online]. Available: https://doi.org/10.1145/3453153

[7] S. Verdu, "Fifty years of shannon theory," IEEE Transactions on Information Theory, vol. 44, no. 6, pp. 2057-2078, Oct 1998.

[8] H. Jegou, M. Douze, and C. Schmid, "Hamming embedding and weak geometric consistency for large scale image search," in Computer Vision - ECCV 2008, D. Forsyth, P. Torr, and A. Zisserman, Eds. Berlin, Heidelberg: Springer Berlin Heidelberg, 2008, pp. 304-317.

[9] Y. Li, S. C. Sundaramurthy, A. G. Bardas, X. Ou, D. Caragea, $\mathrm{X}$. Hu, and J. Jang, "Experimental study of fuzzy hashing in malware clustering analysis," in 8th Workshop on Cyber Security Experimentation and Test (CSET 15). Washington, D.C.: USENIX Association, Aug. 2015. [Online]. Available: https://www.usenix.org/ conference/cset15/workshop-program/presentation/li

[10] B. K. R. Vangoor, V. Tarasov, and E. Zadok, "To FUSE or not to FUSE: Performance of user-space file systems," in 15th USENIX Conference on File and Storage Technologies (FAST 17). Santa Clara, CA: USENIX Association, Feb. 2017, pp. 59-72. [Online]. 
Available: https://www.usenix.org/conference/fast17/technical-sessions/ presentation/vangoor

[11] M. Nelson and J.-L. Gailly, The data compression book. M\&t Books New York, 1996, vol. 2.

[12] S. Camtepe, J. Duda, A. Mahboubi, P. Morawiecki, S. Nepal, M. Pawłowski, and J. Pieprzyk, "Compcrypt-lightweight ans-based compression and encryption," IEEE Transactions on Information Forensics and Security, vol. 16, pp. 3859-3873, 2021.

[13] R. Lyda and J. Hamrock, "Using entropy analysis to find encrypted and packed malware," IEEE Security Privacy, vol. 5, no. 2, pp. 40-45, March 2007.

[14] C. V. Bijitha, R. Sukumaran, and H. V. Nath, "A survey on ransomware detection techniques," in Secure Knowledge Management In Artificial Intelligence Era, S. K. Sahay, N. Goel, V. Patil, and M. Jadliwala, Eds. Singapore: Springer Singapore, 2020, pp. 55-68.

[15] S. Mehnaz, A. Mudgerikar, and E. Bertino, "Rwguard: A real-time detection system against cryptographic ransomware," in Research in Attacks, Intrusions, and Defenses, M. Bailey, T. Holz, M. Stamatogiannakis, and S. Ioannidis, Eds. Cham: Springer International Publishing, 2018, pp. 114-136.

[16] A. Continella, A. Guagnelli, G. Zingaro, G. De Pasquale, A. Barenghi, S. Zanero, and F. Maggi, "Shieldfs: A self-healing, ransomwareaware filesystem," in Proceedings of the 32nd Annual Conference on Computer Security Applications, ser. ACSAC '16. New York, NY, USA: Association for Computing Machinery, 2016, p. 336-347. [Online]. Available: https://doi.org/10.1145/2991079.2991110

[17] J.-Y. Paik, J.-H. Choi, R. Jin, J. Wang, and E.-S. Cho, "A storage-level detection mechanism against crypto-ransomware," in Proceedings of the 2018 ACM SIGSAC Conference on Computer and Communications Security, ser. CCS '18. New York, NY, USA: Association for Computing Machinery, 2018, p. 2258-2260. [Online]. Available: https://doi.org/10.1145/3243734.3278491

[18] S. Baek, Y. Jung, A. Mohaisen, S. Lee, and D. Nyang, "Ssd-insider: Internal defense of solid-state drive against ransomware with perfect data recovery," in 2018 IEEE 38th International Conference on Distributed Computing Systems (ICDCS), 2018, pp. 875-884.

[19] N. Scaife, H. Carter, P. Traynor, and K. R. B. Butler, "Cryptolock (and drop it): Stopping ransomware attacks on user data," in 2016 IEEE 36th International Conference on Distributed Computing Systems (ICDCS), June 2016, pp. 303-312.

[20] A. Kharaz, S. Arshad, C. Mulliner, W. Robertson, and E. Kirda "UNVEIL: A large-scale, automated approach to detecting ransomware," in 25th USENIX Security Symposium (USENIX Security 16). Austin, TX: USENIX Association, Aug. 2016, pp. 757-772. [Online]. Available: https://www.usenix.org/conference/ usenixsecurity16/technical-sessions/presentation/kharaz

[21] J. Gómez-Hernández, L. Alvarez González, and P. García-Teodoro, "R-locker: Thwarting ransomware action through a honeyfile-based approach," Computers \& Security, vol. 73, pp. 389-398, 2018. [Online]. Available: https://www.sciencedirect.com/science/article/pii/ S0167404817302560

[22] B. A. S. Al-rimy, M. A. Maarof, Y. A. Prasetyo, S. Z. M. Shaid, and A. F. M. Ariffin, "Zero-day aware decision fusion-based model for crypto-ransomware early detection," International Journal of Integrated Engineering, vol. 10, no. 6, 2018.

[23] T. Honda, K. Mukaiyama, T. Shirai, T. Ohki, and M. Nishigaki, "Ransomware detection considering user's document editing," in 2018 IEEE 32nd International Conference on Advanced Information Networking and Applications (AINA), 2018, pp. 907-914.

[24] S. Jung and Y. Won, "Ransomware detection method based on contextaware entropy analysis," Soft Computing, vol. 22, no. 20, pp. 6731-6740, may 2018

[25] D. Morato, E. Berrueta, E. Magaña, and M. Izal, "Ransomware early detection by the analysis of file sharing traffic," Journal of Network and Computer Applications, vol. 124, pp. 14-32, 2018. [Online]. Available: https://www.sciencedirect.com/science/article/pii/S108480451830300X

[26] K. Cabaj, M. Gregorczyk, and W. Mazurczyk, "Software-defined networking-based crypto ransomware detection using http traffic characteristics," Computers \& Electrical Engineering, vol. 66, pp. 353-368, 2018. [Online]. Available: https://www.sciencedirect.com/ science/article/pii/S0045790617333542

[27] K. Cabaj and W. Mazurczyk, "Using software-defined networking for ransomware mitigation: The case of cryptowall," IEEE Network, vol. 30, no. 6, pp. 14-20, 2016.

[28] D. F. Netto, K. M. Shony, and E. R. Lalson, "An integrated approach for detecting ransomware using static and dynamic analysis," in 2018 Inter- national CET Conference on Control, Communication, and Computing (IC4), 2018, pp. 410-414.

[29] O. M. K. Alhawi, J. Baldwin, and A. Dehghantanha, Leveraging Machine Learning Techniques for Windows Ransomware Network Traffic Detection. Cham: Springer International Publishing, 2018, pp. 93-106. [Online]. Available: https://doi.org/10.1007/978-3-319-73951-9_5

[30] S. Baek, Y. Jung, A. Mohaisen, S. Lee, and D. Nyang, "Ssd-insider: Internal defense of solid-state drive against ransomware with perfect data recovery," in 2018 IEEE 38th International Conference on Distributed Computing Systems (ICDCS), July 2018, pp. 875-884.

[31] N. Harikrishnan and K. Soman, "Detecting ransomware using gurls," in 2018 Second International Conference on Advances in Electronics, Computers and Communications (ICAECC), 2018, pp. 1-6.

[32] S. Homayoun, A. Dehghantanha, M. Ahmadzadeh, S. Hashemi, R. Khayami, K.-K. R. Choo, and D. E. Newton, "Drthis: Deep ransomware threat hunting and intelligence system at the fog layer," Future Generation Computer Systems, vol. 90, pp. 94-104, 2019. [Online]. Available: https://www.sciencedirect.com/science/article/pii/ S0167739X17328467

[33] J. Baldwin and A. Dehghantanha, Leveraging Support Vector Machine for Opcode Density Based Detection of Crypto-Ransomware. Cham: Springer International Publishing, 2018, pp. 107-136. [Online]. Available: https://doi.org/10.1007/978-3-319-73951-9_6

[34] Z. A. Genç, G. Lenzini, and P. Y. A. Ryan, "No random, no ransom: A key to stop cryptographic ransomware," in Detection of Intrusions and Malware, and Vulnerability Assessment, C. Giuffrida, S. Bardin, and G. Blanc, Eds. Cham: Springer International Publishing, 2018, pp. 234-255.

[35] DIM, "Digital immunity module (dim) demo," 2021. [Online]. Available: https://drive.google.com/file/d/ 1c-x2iC8jTF-DRAYginATWe1yVtewK1VY/view?usp=sharing

[36] - "Digital immunity module (dim) fileless ransomware netwalker demo," 2021. [Online]. Available: https://drive.google.com/file/d/ 1yWPcV4ezV-zqiiEDUe8O7E-tWBUl2Kqz/view?usp=sharing

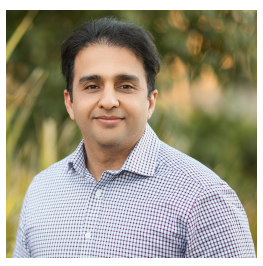

Arash Mahboubi received B.E. degree in computer science (first-class honours) specializing in computer security from the Staffordshire University, Kuala Lumpur, Malaysia in 2012, and received his master's degree in information security from the University Technology Malaysia, Johor Bahru, Malaysia, in 2013. He received his $\mathrm{PhD}$ degree in computer science from the Queensland University of Technology (QUT), Brisbane, Australia, in 2018. From 2016 to 2019 , he was a sessional academic with the School of Electrical Engineering and Computer Science, QUT. Since 2019, he has been a lecturer with the School of Computing and Mathematics, Charles Sturt University, Port Macquarie, New South Wales, Australia. His research interests include computer/mobile malware, ransomware, malware analysis, modeling, and malware epidemic.

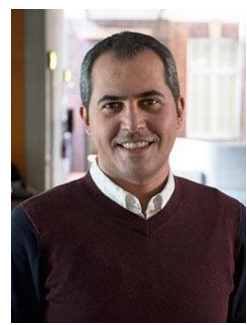

Keyvan Ansari received the B.E. degree in computer engineering from the Sadjad University of Technology, Mashhad, Iran, in 2006, the master's degree (Hons.) in information technology from the University of Newcastle, Callaghan, Australia, in 2009, and the PhD degree in computer science from the Queensland University of Technology (QUT), Brisbane, Australia, in 2014. From 2014 to 2016, Keyvan was an Associate Lecturer with the School of Electrical Engineering and Computer Science, QUT. In 2016, Keyvan joined the University of the Sunshine Coast where he currently undertakes research and lectures in Computer Science and ICT at the School of Science, Technology and Engineering. His research interests include pervasive communications, integrated and distributed IoT systems, and cloud computing. He has co-chaired and has been a member of technical program committees of several international conferences and workshops and has been an editorial board member and a reviewer for several international journals and magazines. 


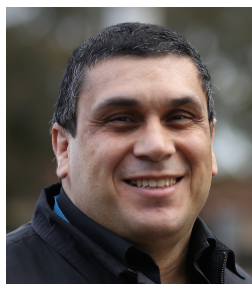

Seyit Camtepe (Senior Member, IEEE) is a principal research scientist and team leader at CSIRO Data61. He received his PhD from Rensselaer Polytechnic Institute in 2007, he was with the Technische Universitaet Berlin as a senior researcher, and with QUT as a lecturer. He was among the first to investigate the security of Android smartphones and inform society for the rising malware threat. His research interests include autonomous security, malware detection and prevention, smartphone security, applied and malicious cryptography, CII security.

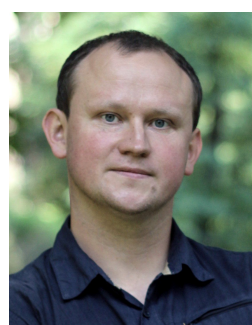

Jarosław Duda (Jarek Duda) is an Assistant Professor at Jagiellonian University. He has education in computer science $(\mathrm{PhD})$, mathematics $(\mathrm{MSc})$ and physics $(\mathrm{PhD})$. He is mainly focused on information theory, statistical analysis, is known from introduction of Asymmetric Numeral Systems.

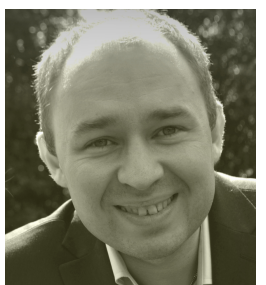

Paweł Morawiecki is an Associate Professor at Institute of Computer Science, Polish Academy of Sciences. From 2017 he is also Head of Cryptography Group at the Institute. His field of expertise includes cryptanalysis and cryptographic algorithms design. Recently he is involved in research at the intersection of security and artificial intelligence, particularly deep neural networks.

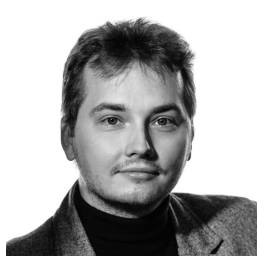

Marcin Pawłowski is a Post-Doc at Institute of Computer Science, Polish Academy of Sciences. He received his $\mathrm{PhD}$ from Warsaw University of Technology where he defended a thesis on the security of Internet of Things. His main research interests lie around design and analysis of security, network and cryptographic protocols especially with applications to distributed systems. He is a consultant working for startups from Blockchain and Internet of Things industries where he crosses scientific theories with real world applications.

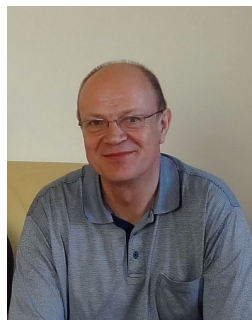

Josef Pieprzyk is a Senior Principal Research Scientist at Data61, CSIRO and a Professor at Institute of Computer Science, Polish Academy of Sciences. His main research interest focus is Cryptology and Information Security and includes design and analysis of cryptographic algorithms (such as encryption, hashing and digital signatures), secure multiparty computations, cryptographic protocols, copyright protection, e-commerce, web security and cybercrime prevention.Dr Pieprzyk is a member of the editorial boards for International Journal of Information Security (Springer), Journal of Mathematical Cryptology (De Gruyter), Open Access Journal of Cryptography (MDPI), International Journal of Applied Cryptography (Inderscience Publishers), Fundamenta Informaticae (IOS Press), International Journal of Security and Networks (Inderscience Publishers) and International Journal of Information and Computer Security (Inderscience Publishers). Dr Pieprzyk published 5 books, edited 10 books (conference proceedings), 6 book chapters, and more than 300 papers in refereed journals and refereed international conferences.

\section{APPENDIX}

A high-level view of the implementation of DIM as a testbed. DIM's pass-through module interacts with three directories, A, B, and C. Directory A contains all files and is not directly accessible to users. Directory B is a shared mounting point (user-level daemon) configured with the Samba protocol and shared with users over the network. Directory $\mathrm{C}$ is located in the user environment to provide access to files over the network. DIM includes a module that keeps all opened files in a temporary directory while reading write buffers to collect file statistics. Once DIM infers that the write buffers are not encrypted, it deletes the temporary directory; otherwise, the temporary folder is used for recovery purposes. As soon as encrypted write buffers are detected, DIM's encoding expansion module is invoked. Note that ransomware executes on a remote user client.

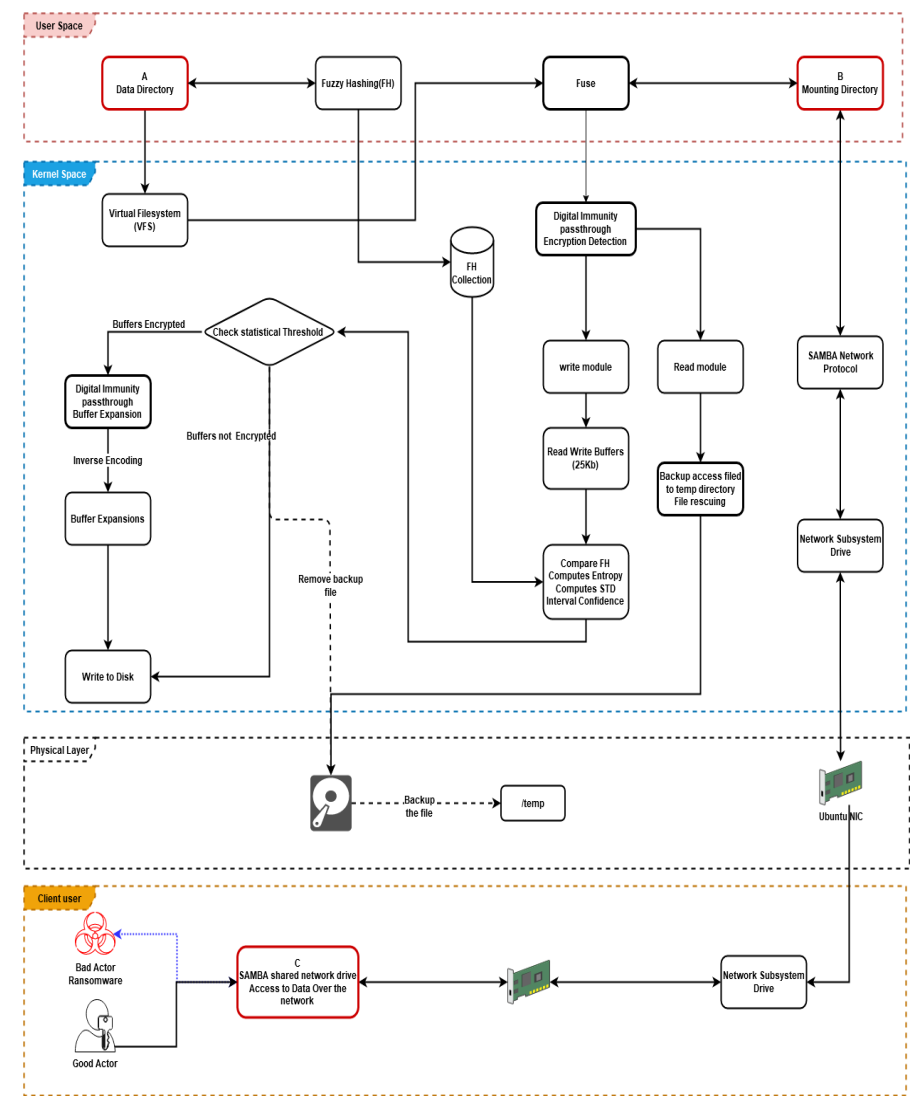

Fig. 7: A high-level view of the implementation of DIM as a testbed. 
TABLE VII: 39 different ransomware were used to validate DIM.

\begin{tabular}{|c|c|c|c|c|c|c|c|c|c|}
\hline \# & SHA256 Hash & Signiture & $\begin{array}{l}\text { Filename } \\
\text { Extension }\end{array}$ & $\begin{array}{c}\text { Entropy } \\
\text { Detection }\end{array}$ & $\begin{array}{c}\text { Max frequency } \\
\text { Occurrence not null }\end{array}$ & Prevention & $\begin{array}{l}\text { Detection } \\
\text { Entropy }\end{array}$ & STD & CI $(99 \%)$ \\
\hline 1 & 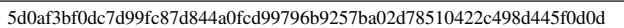 & MedusaLocker & ReadInstruction & $\sqrt{ }$ & $\sqrt{ }$ & $\sqrt{ }$ & 7.9994 & 10.3149 & 3.3527 \\
\hline 2 & Of081ea4e30ca05fc2977235bf239992b17fa9968b58b001990e $4539 f 0899269$ & Avaddon.2 & avdn & $\sqrt{ }$ & $\sqrt{ }$ & $\sqrt{ }$ & 7.9944 & 10.9058 & 3.5448 \\
\hline 3 & 243dff06fc80a049f4fb37292f8b8def0fce29768f345c88ee10699e22b0ae60 & DarkSide & $184 \mathrm{fbc} 3 \mathrm{c}$ & $\sqrt{ }$ & $\sqrt{ }$ & $\sqrt{ }$ & 7.9938 & 11.1970 & 3.6395 \\
\hline 4 & 3dda3ee9164d6815a18a2c23651a53c35d52e3a5ad375001ec824cf532c202e6 & Babuk & babyk & $\sqrt{ }$ & $\sqrt{ }$ & $\sqrt{ }$ & 7.9947 & 10.3319 & 3.3583 \\
\hline 5 & 482e5b77bf6c0c2953e2e8a456a3a072a3f9d5cb35e822e493d062d2372alfc0 & Dharma & ROGER & $\sqrt{ }$ & $\sqrt{ }$ & $\sqrt{ }$ & 7.9945 & 11.0319 & 3.5858 \\
\hline 6 & 48689c986eb553e6a7aeba973501b9660cb1418d4ec3ba9d0511f82799d1422c & Avaddon & $\mathrm{EbBcCCecD}$ & $\sqrt{ }$ & $\sqrt{ }$ & $\sqrt{ }$ & 7.9933 & 10.5478 & 3.4285 \\
\hline 7 & 5837daaf4f7cf7280ec0a749e161015c1de39b35fa26710ce7bb22e352725ed4 & Dharma & aol.com].2020 & $\sqrt{ }$ & $\sqrt{ }$ & $\sqrt{ }$ & 7.9943 & 10.7511 & 3.4945 \\
\hline 8 & 58ccba4fb2b3ed8b5f92adddd6ee331a6afdedfc $755145 \mathrm{e} 0432 \mathrm{a} 7 \mathrm{cb} 324 \mathrm{c} 28053$ & Babuk & babyk & $\sqrt{ }$ & $\sqrt{ }$ & $\sqrt{ }$ & 7.9945 & 11.1845 & 3.6354 \\
\hline 9 & 9a089790e 04683ebf37d9746e0284322f59c46eef2a86cc231839482f323e871 & Babuk & babyk & $\sqrt{ }$ & $\sqrt{ }$ & $\sqrt{ }$ & 7.9934 & 11.0577 & 3.5942 \\
\hline 10 & c34d0660da24b48480de58aaa394bd27f5a5b1ed9249d897ca4dde70312a87ec & Makop & moloch & $\sqrt{ }$ & $\sqrt{ }$ & $\checkmark$ & 7.9946 & 10.6242 & 3.4533 \\
\hline 11 & dffefbde27442b9095388b 1871 ffdc101c430b9a814138be4f962328a5b73fde & Makop & usagoo & $\sqrt{ }$ & $\sqrt{ }$ & $\sqrt{ }$ & 7.9942 & 10.2064 & 3.3175 \\
\hline 12 & eacbf729bb96cf2eddac62806a555309d08a705f6084dd98c7cf93503927c34f & Nefilim & OFFWHITE & $\sqrt{ }$ & $\sqrt{ }$ & $\sqrt{v}$ & 7.9947 & 10.6832 & 3.4725 \\
\hline 13 & ed01ebfbc9eb5bbea545af4d01bf5f1071661840480439c6e5babe8e080e41aa & WannaCryptor & WNCRY & $\sqrt{ }$ & $\sqrt{ }$ & $\sqrt{ }$ & 7.9947 & 10.2644 & 3.3363 \\
\hline 14 & fa722d0667418d68c4935e1461010a8f730f02falf595ee68bd0768fd5dlf8bb & HelloKitty & crypted & $\sqrt{ }$ & $\sqrt{ }$ & $\sqrt{ }$ & 7.9938 & 10.9654 & 3.5642 \\
\hline 15 & fb54alb85ab37cdee346e06cf716cbe0b071f4833020823595f3b69614c5446e & Xorist & locks & $\sqrt{ }$ & $\sqrt{ }$ & $\sqrt{ }$ & 7.9946 & 9.6708 & 3.1433 \\
\hline 16 & e8a091a84dd2ea7ee $429135 f f 48 e 9 f 48 f 7787637 \mathrm{ccb} 79 f 6 c 3 e b 42 f 34588 \mathrm{bc}$ & Maze & random & $\sqrt{ }$ & $\sqrt{ }$ & $\sqrt{ }$ & 7.9943 & 10.9694 & 3.5655 \\
\hline 17 & 6e9060d56e669658b059f25a05f37f4d266658fece 36afdb564536607fd9570b & Matrixran & CORE & $\sqrt{ }$ & $\sqrt{ }$ & $\sqrt{ }$ & 7.9942 & 10.0732 & 3.2741 \\
\hline 18 & 750984dff0d13260e17e9bb1a3482f1bae834d6e0de1bcd199028748a9f998dc & GlobeImposter & $\begin{array}{l}\text { Globeimposter- } \\
\text { Alpha865qqz }\end{array}$ & $\sqrt{ }$ & $\sqrt{ }$ & $\sqrt{ }$ & 7.9903 & 11.4079 & 3.7080 \\
\hline 19 & 0d7ed584dd1ae3cc071ad1b2400a5c534d19206be7a98a6046959a7267c063a1 & Netwalker & sha256 & $\sqrt{ }$ & $\sqrt{ }$ & $\sqrt{ }$ & 7.9934 & 10.6136 & 3.4499 \\
\hline 20 & 3f6e996ee4a40d2d19b648669d9146562627359626239324937a5c75f8030824 & SunCrypt & sha256 & $\sqrt{ }$ & $\sqrt{ }$ & $\sqrt{ }$ & 7.9926 & 11.3203 & 3.6796 \\
\hline 21 & d85188c58acb395ae88ad2be1f48044090eb03f125c97692c20787b933bbbd1a & Snatch & aulmhwpbpzi & $\sqrt{ }$ & $\sqrt{ }$ & $\sqrt{ }$ & 7.9930 & 11.0849 & 3.6030 \\
\hline 22 & 5d0af3bf0dc7d99fc87d844a0fcd99796b9257ba02d78510422c498d445f0d0d & RanzyLocker & RANZYLOCKED & $\sqrt{v}$ & $\sqrt{ }$ & $\sqrt{ }$ & 7.9940 & 10.2473 & 3.3308 \\
\hline 23 & 6931b124d38d52bd7cdef48121fda457d407b63b59bb4e6ead4ce548f4bbb971 & Darkside & $463 \mathrm{f} 7015$ & $\sqrt{ }$ & $\sqrt{ }$ & $\sqrt{ }$ & 7.9922 & 11.5609 & 3.7577 \\
\hline 24 & d786355c1b3dc741103873aed46d8ffa3430d113a27482f37f3ffc7c978747f6 & FuxSocy & fse_ $4 \mathrm{~b} 270244$. . & $\sqrt{ }$ & $\sqrt{ }$ & $\sqrt{v}$ & 7.9938 & 10.3384 & 3.3604 \\
\hline 25 & c14987c4c6fc2de2cac 43355964465 d $7611652 \mathrm{e} 29 \mathrm{f} 699 \mathrm{~d} 64 \mathrm{fa} 292399 \mathrm{f} 526 \mathrm{c} 103$ & Wrui & wrui & $\sqrt{ }$ & $\sqrt{ }$ & $\sqrt{v}$ & 7.9933 & 10.7011 & 3.4783 \\
\hline 26 & f4656a9af30e98ed2103194f798fa00fd1686618e3e62fba6b15c9959135b7be & PS1.NETWALKER.B & b79e2e & $\sqrt{ }$ & $\sqrt{ }$ & $\sqrt{ }$ & 7.9944 & 10.7411 & 3.5782 \\
\hline
\end{tabular}

TABLE VIII: Ransomware that do not attack the shared drive.

\begin{tabular}{|c|c|c|c|c|c|}
\hline \# & Signiture & Signiture & Filename Extension & Attacking shared drive & Comments \\
\hline 27 & 8d2f2ee24882afe 11 f50e3d6d9400e35fa66724b321cb9f5a246baf63cbc 1788 & CryptoLocker & betarasite & - & $\begin{array}{l}\text { Regardless of original file size, ransomware crupte all files to } 256 \text { bytes. } \\
\text { significant flaw it encrypts data with the wrong cipher and overwrites data with } 256 \text { bytes. } \\
\text { https://www.bugsfighter.com/remove-parasite-ransomware-and-decrypt-parasite-betarasite-or-paras Ite-files }\end{array}$ \\
\hline 28 & $3169 \mathrm{c} 344 \mathrm{~b} 627318 \mathrm{~d} 17 \mathrm{c} 9 \mathrm{a} 92726460 \mathrm{ea} 45 \mathrm{~b} 196 \mathrm{ccbf} 0 \mathrm{f} 1 \mathrm{abe} 246 \mathrm{faf} 7069 \mathrm{e} 0701 \mathrm{a} 2$ & covid-21 & - & - & Restarting the system after locking the victim hard drive. \\
\hline 29 & $01157 \mathrm{c} 3 \mathrm{e} 056 \mathrm{~d} 2040250598 \mathrm{bc} 9 \mathrm{~b} 4 \mathrm{aac} 8 \mathrm{~b} 4 \mathrm{ad} 8 \mathrm{~b} 7 \mathrm{f2c5} 595381 \mathrm{~d} 320290 \mathrm{dd} 79 \mathrm{~b} 8317 \mathrm{~d}$ & Winlock (Corana) & - & - & Restarting the system after locking the victim hard drive. \\
\hline 30 & 6a5090762c6058bc223e37e89f53832faad80995e3c5ed7e59ed9f5a5e604e 47 & Thanos & crypted & $\sqrt{ }$ & - \\
\hline 31 & 8e775324fc69a677394cf6d079d1d45bf53af10acd683bda53e5f86a8a192393 & TeslaCrypt & ololqq & $\sqrt{v}$ & _ \\
\hline 32 & 9fa3a004576f357b5174dd1c29ef7d13005d996d5f9fb4b86d6d978d1a4a84ae & Sodinokibi & 99 un1 & 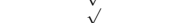 & _ \\
\hline 33 & b5466ce462df16b3a29f22192b1291d70479cacf35bd5e937f355b2567da948fe & RagnarLocker & 93F28.thor & $\mathrm{v}$ & - \\
\hline 34 & c97d9bbc80b573bdeeda3812f4d00e5183493dd0d5805e2508728f65977dda 15 & LockerGoga & locked & $\sqrt{v}$ & - \\
\hline 35 & cc0372ablaba3269d4aab5abaeofocb25138302dc7fa36db19fe 7e la9ad2e2d9 & Sodinokibi & atjyzypdo & $\sqrt{v}$ & - \\
\hline 36 & $\mathrm{db} 665 \mathrm{f} 26 \mathrm{dbc} 4 \mathrm{ca} 92 \mathrm{~d} 326 \mathrm{f} 2 \mathrm{cb} 98 \mathrm{faafb} 9 \mathrm{e} 84 \mathrm{~d} 404346 \mathrm{~b} 201 \mathrm{~cd} 88 \mathrm{bec} 91 \mathrm{ce} 4206 \mathrm{~b} 2$ & WastedLocker & howto_seccrypt & $\sqrt{v}$ & - \\
\hline 37 & e3bf41de3a7edf556d43b6196652aa036e48a602bb3f7c98af9dae992222a8eb & WastedLocker & eswasted & $\sqrt{v}$ & - \\
\hline 38 & 2b9838da7edb0decd32b086e47a31e8f5733b5981ad8247a2f9508e232589bff & DoejoCrypt & CRYPT & $\sqrt{ }$ & - \\
\hline 39 & $33352 \mathrm{a} 38454 \mathrm{cfc} 247 \mathrm{bc} 7465 \mathrm{~b}$ f177f5f97d7fdobd220103d4422c8ec45b4d3d0e & Cuba & cuba & $\sqrt{ }$ & Cuba has merged with the Hancitor malware in order to spread. \\
\hline
\end{tabular}

